\section{Introducing conservation marketing: why should the devil have all the best tunes?}

The choices we make every day have created lifestyles that are a key driver of all major threats to the environment. This makes influencing human behaviour the ultimate challenge for a conservationist.

This challenge is not unique to conservation science. In the commercial sector there have been decades of research on how to influence consumers. This knowledge has not gone unnoticed in the non-profit sector, and since the 1970 social marketing (i.e. the use of marketing principles for social good) has grown in popularity. The use of marketing principles has also expanded in areas such as public health but has remained relatively scarce in biodiversity and environmental conservation.

Conservationists have largely been uneasy about relying on the same dark arts used to sell products such as cigarettes and alcohol, leaving untapped the potential of using marketing to drive the adoption of environmentally friendly behaviours. But shouldn't the ethic behind the need to conserve nature be powerful enough to drive change? In August more than 2,000 conservationists from 90 countries gathered in Montpelier, France, for the 27th International Congress of Conservation Biology, organized by the Society for Conservation Biology (SCB). Amongst the 60 symposia there was a newcomer in the social sciences: the first session dedicated to the use of marketing tools in biodiversity conservation. This session built on the success of an event at the International Marine Conservation Congress in Glasgow in 2014.

The symposium brought together academics and practitioners from the commercial and non-profit sectors to discuss how marketing can best support conservation. The topics covered were diverse, from wildlife trade and marine conservation to the use of celebrity endorsements. Our goal was to reframe what marketing means in the context of conservation, away from the perception of being a dark art and towards its adoption as a new path for more effective behaviour change. The symposium was well attended, with those present exceeding the seating capacity of the room and revealing a yet untapped interest in conservation marketing.

To address this demand and interest the Conservation Marketing and Engagement Working Group (ConsMark), which will function within the SCB, has been formed. The group defines conservation marketing as 'the ethical application of marketing strategies, concepts and techniques to influence attitudes, perceptions and behaviours of individuals, and ultimately societies, with the objective of advancing conservation goals'. ConsMark has several objectives. One is to promote the use of marketing techniques and strategies to tackle environmental issues. Another is to improve access for conservation practitioners to marketing tools and to build capacity for their effective use. With the support of the SCB, ConsMark now has a website and a mailing list open to non-SCB members, where you can find out more about the group and its work (http://conbio. org/groups/working-groups/conservation-marketingworking-group).

We urge all who see the adoption of more environmentally friendly behaviours as a key to address the challenge of conserving biodiversity to join our efforts to mainstream the use of marketing tools and principles.

Diogo Verissimo Department of Economics, Georgia State University, Atlanta, Georgia, USA, and Rare, Arlington, Virginia, USA. E-mail verissimodiogo@gmail.com

EMMA MCKINLEY Business School, University of Chichester, Bognor Regis, UK

\section{Wildlife forensic science in the investigation of poaching of vicuña}

Chilean authorities, with the help of the U.S. National Fish and Wildlife Forensic Laboratory, are currently developing crime scene investigation techniques and forensic protocols to combat an increase in poaching of the vicuña Vicugna vicugna occurring on the Andean altiplano. The techniques and protocols are being shared with other countries in the vicuña's range (Chile, Peru, Bolivia, Argentina and Ecuador).

Over 5,000 vicuñas have been killed in recent years for their valuable fur, the black market value of which is USD 100-200 per $\mathrm{kg}$. The corpses of 1,823 skinned vicuñas were found in Peru and 3,289 in Bolivia during 2008-2015, 149 in Argentina during 2012-2013 and 51 in Chile during the first 4 months of 2014. An additional 337 pelts were confiscated from smugglers caught at international borders during 2014-2015. These figures are probably underestimates of losses in a problem that is increasingly difficult to monitor and control. The vicuña was on the verge of extinction in the 1960 s and 1970s, mostly as a result of poaching, but recovered as a result of conservation efforts across its range. The current poaching is, however, better organized: hunters use motor vehicles and long-range firearms, and are bold enough to shoot at law enforcement officers.

Coordinated action between regulatory and law enforcement institutions in Chile has resulted in increased patrols in high-risk areas and an apparent decrease in local poaching. In addition, officials working for the National Forest Core, Agriculture and Farming Service, Chilean Police and Chilean Investigative Police, and judges, prosecutors and university professors have been trained in crime scene investigation techniques at the U.S National Fish and Wildlife Forensic Laboratory. This has allowed Chilean 